\title{
CLIMA URBANO E EVOLUÇÃO DA TEMPERATURA ESTIVAL EM LISBOA NO SÉCULO XX. TENDÊNCIA, NÚMERO DE NOITES QUENTES E AMPLITUDE TÉRMICA DIÁRIA*
}

\author{
Paulo Pereira ${ }^{1}$ \\ LILIANE MORAIS ${ }^{2}$
}

\begin{abstract}
Resumo - Existem muitas evidências de que as cidades influenciam o clima local, particularmente no que diz respeito à temperatura, ocasionando uma "ilha de calor urbano", com impactes negativos no conforto bioclimático e na saúde humana. Durante o século transacto, sobretudo a partir de 1980, ocorreu um aumento da temperatura dos meses de Verão (Junho, Julho, Agosto e Setembro, JJAS), particularmente no que diz respeito à temperatura mínima média. Verificou-se igualmente um aumento de noites "quentes" (de temperatura mínima superior a $20^{\circ} \mathrm{C}$ ), em especial em Junho e Setembro e uma diminuição significativa da amplitude térmica diurna, nomeadamente nos últimos anos do século XX. Estes factos poderão ser devidos não só ao efeito urbano, mas também ao aquecimento global.
\end{abstract}

Palavras-chave: Conforto bioclimático, saúde humana, ilha de calor urbana, aquecimento global, noites quente, amplitude térmica diurna.

\begin{abstract}
Urban Climate AND SUMMER TEMPERATURes IN Lisbon DURING THE $20^{\text {TH }}$ CENTURY. TRENDS, NUMBER OF HOT NIGHTS AND DIURNAL TEMPERATURE RANGE. There is ample evidence of the cities' effects upon the local climate and, in particular, upon temperature, by giving rise to the so-called "urban heat island" (UHI), which has a strong negative impact upon human bioclimatic comfort and health.

In this study, temporal evolution of summer (June, July, August and SeptemberJJAS) temperature trends in Lisbon are studied. During the course of the $21^{\text {st }}$ Century, a temperature increase was found to have occurred in this city (most notably since 1980 and particularly as regards the mean minimum temperature), alongside an increase in the number of nights in which the temperature has reached values higher than $20^{\circ} \mathrm{C}$ and a reduction of the diurnal temperature range, all of which can be attributed to urban growth and/or global warming.
\end{abstract}

* Recebido: 4/12/2006. Revisto: 20/05/2007 e 9/07/2007. Aceite: 24/07/2007.

1 Investigador do Grup de Recerca Ambiental Mediterrànea (GRAM), Departament de Geografia Física i Anàlisi Geográfica Regional, Facultat de Geografia i Història, Universitat de Barcelona. E-mail: daviolas@portugalmail.pt

2 Geógrafa. Câmara Municipal de Lisboa. E-mail: lilianemorais7@hotmail.com 
Key words: Bioclimatic comfort, human health, Urban heat island, global warming, hot nights, diurnal thermal range.

Résumé - LE CLIMAT URBAIN ET L’ÉVOLUTION DE LA TEMPÉRATURE A LiSBONNE PENDANT LE XX ${ }^{\mathrm{E}}$ SIĖCle. TendANCE, NOMBRE DE NUits CHAUdeS ET AMPLiTUde THERMIQUE JOURNALIÈRE. Il est prouvé que les villes affectent le climat local, surtout en ce qui concerne les températures, en créant un îlot de chaleur urbain, lequel diminue le confort bioclimatique des habitants. Pendant le $\mathrm{XX}^{\mathrm{e}}$ siècle (particulièrement depuis 1980), la température des mois de juin, juillet, août et septembre a augmenté à Lisbonne, surtout en ce qui concerne la moyenne des minimums. Ceci peut être dû soit à l'îlot de chaleur urbain, soit au « réchauffement global ».Le nombre de nuits dont la température est supérieure à $20^{\circ} \mathrm{C}$ a aussi augmenté, ainsi que l'amplitude thermique journalière (DTR), en conséquence de la croissance urbaine.

Mots-clés: Confort bioclimatique, santé humaine, îlot de chaleur urbain, réchauffement global, nuits chaudes, amplitude thermique journalière.

\section{INTRODUÇÃO}

Está plenamente comprovado que a cidade apresenta uma temperatura mais elevada que os arredores (Lowry, 1976; Oke, 1987).

De todas as alterações induzidas pela presença de uma cidade, o aumento de temperatura é a mais evidente. Este fenómeno tem o nome de ilha de calor urbano (IC) e é essencialmente nocturno, desenvolvendo-se em condições específicas, principalmente em situações de vento fraco e céu limpo. Pode variar na intensidade, configuração e localização espacial do seu máximo térmico. Apresenta padrões distintos consoante a altura do dia, situação meteorológica, localização da cidade no globo e características morfológicas. Existem três tipos de IC: de superfície (surface heat island), de atmosfera inferior (canopy layer heat island) e superior (boundary layer heat island) (Moreno e Martin Vide, 2002; Solecki, et al., 2002; Vogt e Oke, 2003; McClatchey, 2005; Jin et al., 2005).

A IC pode ter impactes directos ou indirectos na saúde e no conforto térmico humano. Consideram-se positivos ou negativos consoante a estação do ano e a posição da cidade em latitude. Durante o Inverno, nos aglomerados urbanos de latitudes médias, reduz o consumo de energia e os óbitos como consequência do frio; no entanto, durante o Verão verifica-se um aumento do stress térmico e da mortalidade dos mais idosos (Landsberg, 1973; Taha, 1997; Fukuoka, 1997). Esta situação é particularmente grave em episódios de vagas de calor, que aumentam os efeitos da poluição, nomeadamente do ozono troposférico $\left(\mathrm{O}_{3}\right)$, nocivo para a saúde humana (Bosch, 2003; Shar e Jendritzky, 2004; Epstein e Rogers, 2004).

Um dos principais factores que contribui para a IC é a diminuição do albedo (fig. 1a), que atinge valores baixos nas cidades, devido ao elevado grau de radiação interceptada ou absorvida, influenciando o balanço radiativo (Bretz et al., 1998; Chimklai et al., 2004). Em média, o albedo numa cidade varia entre 0,10 e 0,20, mas pode ultrapassar largamente estes números, além de variar dentro da própria cidade 
(fig. 1b). Os centros urbanos do Norte de África apresentam um albedo médio de 0,30-0,45, ao passo que nos europeus e americanos observa-se uma reflexão muito menor, 0,15-0,30 (Taha, 1997).

Investigações efectuadas por Jin et al. (2005) indicam que as cidades localizadas entre $30^{\circ}-65^{\circ}$ de latitude exibem um albedo inferior entre 2 e $5 \%$ em relação aos seus arredores. A diminuição da reflexão deve-se à cor e ao tipo dos materiais de construção e à morfologia da superfície urbana. Estudos realizados por Bretz et al. (1998) em Sacramento, Califórnia, demonstram que aproximadamente $20 \%$ das $96 \mathrm{mi}^{2}$ da cidade apresentam telhados escuros e $10 \%$ são ocupados por pavimentos negros. Estima-se que se estas superfícies fossem alteradas o albedo aumentaria em $18 \%$. Segundo Prado e Ferreira (2005) o tipo de materiais de construção dos telhados é uma das variáveis mais importantes na sua absorção de calor.

Outro problema relacionado com a IC é a sua possível contribuição para o recente aumento de temperaturas a nível global. A questão ainda não é consensual (Sala et al., 2000; Parker, 2004). Investigações realizadas por Kalnay e Cai (2003) indicam que, durante o século XX, a urbanização e as alterações no uso do solo foram responsáveis pelo acréscimo de $0,27^{\circ} \mathrm{C}$ da temperatura nos Estados Unidos. No entanto, muitas discussões existem sobre estes resultados. Parker (2004) afirma que o aumento da temperatura a nível global não se deve ao efeito urbano (Alcoforado e Andrade, 2007, no prelo). No entanto, é incontestável que as cidades são particularmente vulneráveis ao efeito do aquecimento global.

Outra consequência importante dos centros urbanos no clima é a diminuição da amplitude térmica diária (ATD), que está relacionada com a maior absorção de calor pelos materiais pétreos da cidade. Observa-se que o aumento da temperatura média mínima, $\left(\mathbf{T}_{\text {min }}\right)$ é mais acentuado que o da temperatura média máxima $\left(\mathbf{T}_{\text {max }}\right)$ (Easterling et al., 1997; Stone e Weaver, 2002; Vose et al., 2004).

Este trabalho tem duas principais finalidades: 1) estudar a evolução térmica estival em Lisboa (em Junho, Julho, Agosto e Setembro, JJAS), altura em que o stress tér-

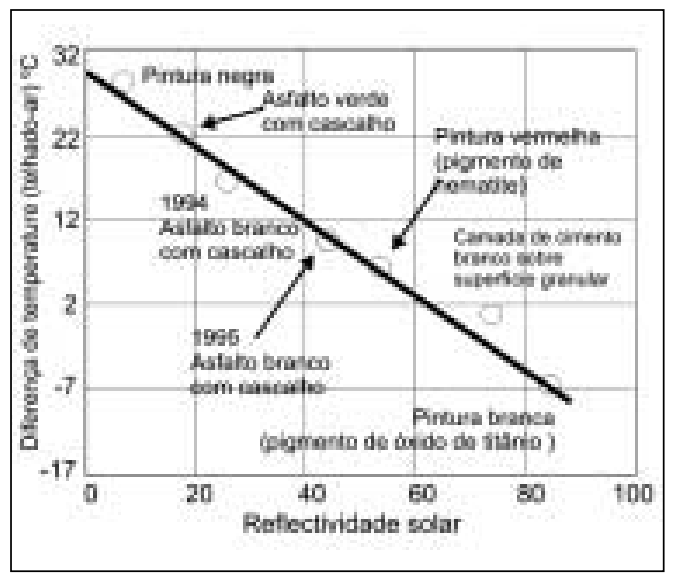

Fig.1a) - Albedo dos diferentes materiais (Fonte: http://eetd.lbl.gov/HeatIsland/CoolRoofs). Fig. 1a) - Albedo of the different materials (Source: http://eetd.lbl.gov/HeatIsland/CoolRoofs).

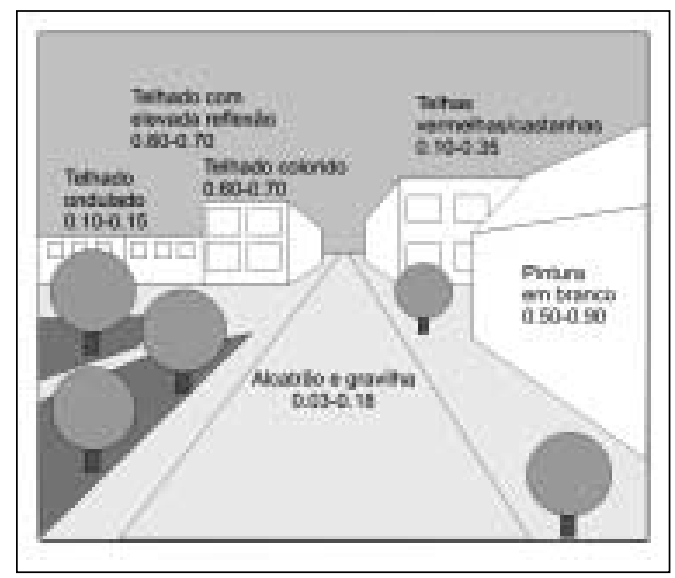

Fig.1b) - Variação do albedo na cidade (Adaptado de EPA, 2003).

Fig. 1b) - Albedo's values in town (Adapted from EPA, 2003). 
mico devido ao calor é mais elevado, ao longo do século XX (1901/2001) e em diferentes períodos (1951/2001 e 1971/2001); e 2) observar a evolução e comportamento do número de noites quentes, com temperaturas acima dos $20^{\circ} \mathrm{C}$, e da ATD entre 19512001 e em diferentes períodos (1951/1975 e 1976/2001).

\section{DADOS E MÉTODOS APLICADOS}

\section{Dados}

As temperaturas são registadas na estação de Lisboa/Geofísico, a $38^{\circ} 43^{\prime} \mathrm{N}$, e $09^{\circ} 09^{\circ} \mathrm{W}$ e de $77 \mathrm{~m}$ de altitude. É a que apresenta a série de dados mais longa e fiável em Lisboa. Estão disponíveis em http://eca.knmi.nl/ (Klein Tank et al., 2002) do European Climate Assesment, que se encontram já homogeneizados.

\section{Métodos aplicados}

Para o presente trabalho, foi estudada a evolução das temperaturas média mínima, $\mathbf{T}_{\text {min }}$, média, $\mathbf{T}_{\text {med }}$, e média máxima, $\mathbf{T}_{\max }$, ao longo do século XX - 1901/2001 - observando-se os desvios à média de cada ano, relativamente à normal 1961/1990, durante os meses de JJAS. Para entender o seu comportamento durante o século transacto, procedeu-se ao exame da evolução em três períodos distintos, 1901/2001, 1951/2001 e 1971/2001; neste caso não a partir dos desvios à média, mas analisado as temperaturas absolutas. Aqui, aplica-se o estudo de algumas estatísticas descritivas, como a Média $\left(\mathbf{M}_{\mathrm{d}}\right)$, o Mínimo $\left(\mathbf{M}_{\mathrm{m}}\right)$, o Máximo $\left(\mathbf{M}_{\mathbf{x}}\right)$, a Variância $\left(\mathbf{V}_{\mathrm{ar}}\right)$, o Desvio Padrão $\left(\mathbf{D}_{\mathrm{p}}\right)$ e a análise dos coeficientes de Assimetria - Skewness - $\left(\mathbf{S}_{\mathbf{k}}\right)$ e Achatamento - Kurtosis $\left(\mathbf{K}_{u r}\right)$. Através do $\mathbf{S}_{\mathbf{k}}$ pode observar-se a distribuição dos valores relativamente à $\mathbf{M}_{\mathbf{d}}$. Se o coeficiente for negativo, significa que os valores da série estão maioritariamente acima da $\mathbf{M}_{\mathrm{d}}$, ao passo que se for negativo é o inverso. A $\mathbf{K}_{\mathrm{ur}}$ permite-nos observar a posição dos valores ao longo da série. Se o coeficiente for negativo, regista-se uma maior heterogeneidade, observando-se o contrário quando é positivo. Para estimar a normalidade das distribuições, foi empreendido o teste de Shapiro-Wilk (ShW) (Shapiro e Wilk, 1965) e o respectivo $p$-value - assumindo-se para valores de $p<0.05$ a distribuição como não normal; quanto maior for o resultado do teste $S h W$ e o $p$-value, maior a normalidade da distribuição - também aplicado em outros estudos que se debruçam sobre tendências climáticas (Scherrer et al., 2006).

Com o objectivo de avaliar os valores extremos mais elevados da $\mathbf{T}_{\min }, \mathbf{T}_{\operatorname{med}}$ e $\mathbf{T}_{\max }$, ordenaram-se as temperaturas - e os respectivos anos - da mais elevada para a mais baixa, isolando em seguida os dez anos mais quentes de toda a série de 101 anos - que correspondem aos valores superiores ao $9 .^{\circ}$ decil, uma técnica também aplicada em outros estudos que analisam os extremos de distribuição da temperatura (Frich et al., 2002; Moberg e Jones, 2005; Klein Tank et al., 2005). Subsequentemente, procurou-se nos valores seleccionados o total de Verões com temperaturas elevadas a partir de 1980 e de 1990 que, segundo outros estudos a nível global, parecem ser os mais quentes de todo o século XX (Bradley, 2000a e b).

$\mathrm{Na}$ análise do número de noites quentes, com temperatura mínima superior a $20^{\circ} \mathrm{C}$ que, segundo Epstein e Rogers (2004) influenciam consideravelmente o con- 
forto bioclimático e, à semelhança do que acontece com o estudo das temperaturas no século XX, são examinadas as mesmas estatísticas descritivas, durante o período 1951/2001. Além de se analisarem os últimos 50 anos do século transacto, procedeu-se a sua divisão (1951/1975 e 1976/2001) no sentido de averiguar o seu comportamento e se existem diferenças significativas no seu padrão, aplicando o teste t-student. Por último, são descriminadas as contribuições de cada mês para o total de noites quentes entre 1951-2001, mas também a ocorrência de noites com temperaturas $>20^{\circ} \mathrm{C}$ em JJAS entre 1951/1975 e 1976/2001. A aplicação do teste $S h W$ determinou estas distribuições como não normais, empreendendo-se assim, em alternativa ao teste $t$-student, o teste não paramétrico Wilcoxon matched pairs test que faz uma comparação entre as medianas. A mesma metodologia utilizada no número de noites quentes foi aplicada no estudo da evolução da ATD, analisando-se posteriormente a relação entre ambas, onde se observa a posição ou não de todos os valores dentro do perímetro da elipse normal a 99\%, para identificar a presença de outliers ou valores extremos.

As análises das estatísticas descritivas de todas as temperaturas foram efectuadas no software Statistica 6.0 (Statsoft inc.).

\section{CARACTERÍSTICAS GERAIS DO CLIMA DE LISBOA E DA SUA IC}

Lisboa é uma cidade com clima mediterrâneo (Csa) segundo a classificação de Köppen. Segundo a normal 1961/1990 - estação de Lisboa/Geofísico - apresenta uma $\mathbf{T}_{\max }$ anual de $20,8^{\circ} \mathrm{C}$, uma $\mathbf{T}_{\text {med }}$ anual de $16,8^{\circ} \mathrm{C}$ e uma $\mathbf{T}_{\min }$ anual de $12,6^{\circ} \mathrm{C}$. O total de precipitação anual é de $750,7 \mathrm{~mm}$ e concentra-se maioritariamente entre Novembro e Fevereiro (http://www.igidl.ul.pt/climat.htm). Os ventos, durante o Verão têm origem maioritariamente no quadrante Norte $(76 \%)$, ao passo que na estação invernal têm uma proveniência mais heterogénea (Alcoforado e Lopes, 2003).

A IC de Lisboa tem uma intensidade média durante a noite no Verão e no Inverno comparada com os arredores de $2,5^{\circ} \mathrm{C}$. No entanto, a localização dos espaços mais quentes difere consoante a acção do vento. Em situações de vento forte, a maior intensidade é observada no centro da cidade - mais abrigado - onde as altitudes são mais baixas (Alcoforado e Andrade, 2006).

\section{RESULTADOS}

\section{Evolução da $\mathbf{T}_{\text {min }}, \mathbf{T}_{m e d}$ e $\mathbf{T}_{\text {max }}$ estival (JJAS) durante o século $\mathbf{X X}$}

Todas as temperaturas em análise demonstram uma evolução no sentido de um aquecimento (fig. 2). Apesar das oscilações observadas em relação à normal 1960/91, a $\mathbf{T}_{\text {min }}$ regista um aumento progressivo até finais dos anos 30 , quando sofre uma diminuição até princípios da década de 40 , estabilizando até ao início da década de 80 para posteriormente mostrar uma subida acentuada. Relativamente à $\mathbf{T}_{\text {med }}$ observa-se uma maior variabilidade na sua dinâmica. A sua evolução desenvolveu-se ao longo de três fases. Na primeira, entre 1901/1946, regista-se um aquecimento - intercalado por um pequeno período frio durante os anos 30 - seguido de um arrefecimento entre 1946/1975. Nos últimos 25 anos do século XX, verificou-se uma subida importante da $\mathbf{T}_{\text {med }}$, ape- 
sar de mostrar menor magnitude do que a verificada para a $\mathbf{T}_{\min }$. $\mathrm{O}$ comportamento da $\mathbf{T}_{\max }$ foi marcado por um grande aquecimento até aos anos 50 - principalmente entre 1941/1951 - registando-se subsequentemente um declínio até finais dos anos 70. A partir da década de 80 , observa-se um incremento, embora não tão pronunciado como nas restantes temperaturas.
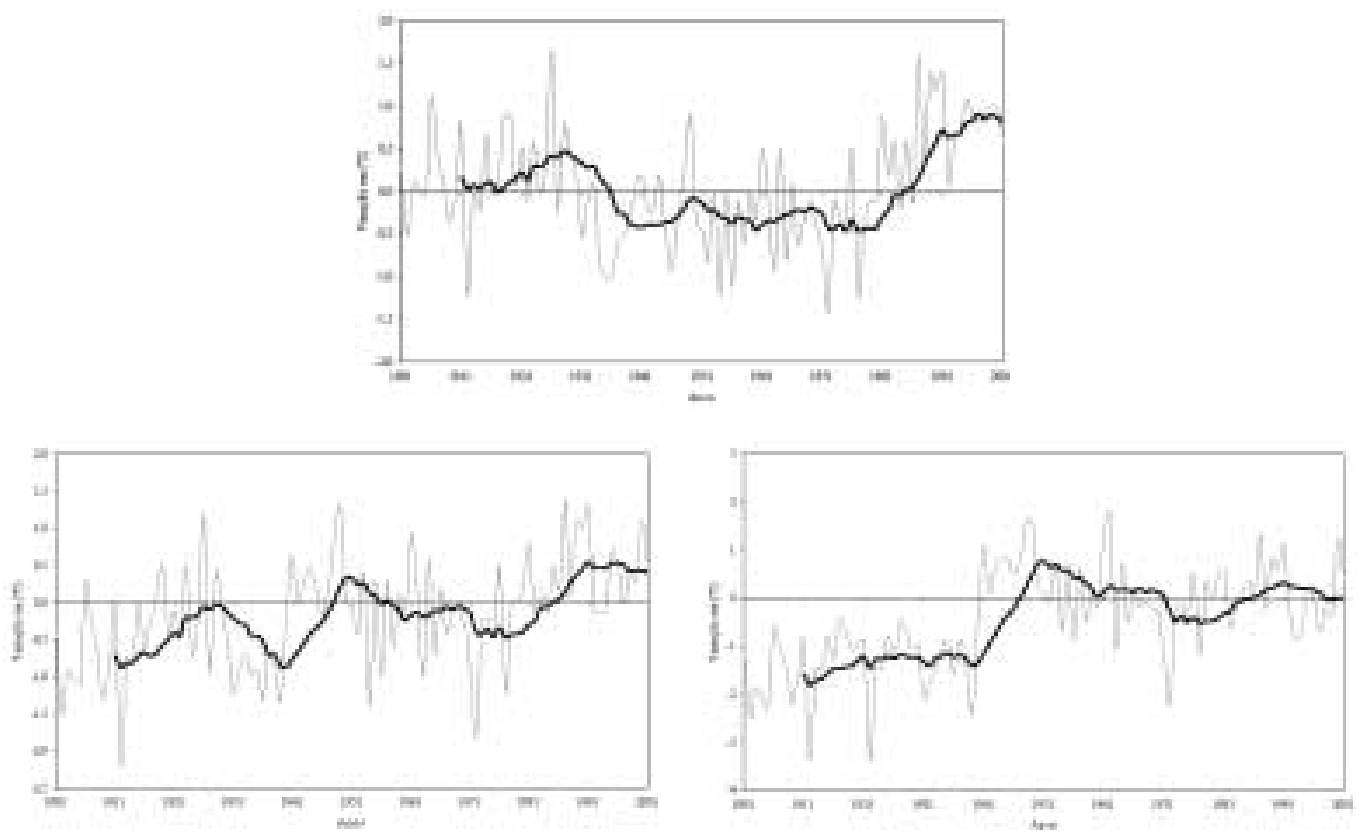

Fig. 2 - Desvios da temperatura à normal estival 1961/90 (JJAS) em Lisboa durante o século XX. A linha a negrito corresponde à média móvel de onze anos.

Fig. 2 - Temperature deviation, to the 1961/90 summer normal (JJAS) in Lisbon, during the XX century. The bold line corresponds to the moving averages (period: eleven years).
a) $\mathrm{T}_{\min }$;
b) $\mathrm{T}_{\text {med }}$;
c) $\mathrm{T}_{\max }$

Observando os ritmos de aquecimento durante o século XX em Lisboa (quadro 1), verifica-se que, entre 1901/2001, a $\mathbf{T}_{\max }$ foi a que sofreu um aumento mais substancial, muito por culpa do grande incremento nos primeiros 50 anos. Nos últimos 51 e 31 anos a temperatura que subiu mais foi a $\mathbf{T}_{\min }$. Neste último período, todas as temperaturas apresentaram um aumento, nomeadamente a $\mathbf{T}_{\min }$ e a $\mathbf{T}_{\text {med }}$.

Analisando todo o século XX (quadro 2), foi a $\mathbf{T}_{\min }$ que apresentou um menor desvio à média em comparação com as restantes. Relativamente a esta, os valores da $\mathbf{T}_{\text {med }}$ e $\mathbf{T}_{\max }$ apresentam uma distribuição negativa no $\mathbf{S}_{\mathrm{k}}$, ou seja, uma tendência para os valores extremos positivos localizando-se a maior parte das ocorrências acima da $M_{d}$, principalmente na segunda. Relativamente à $\mathbf{T}_{\min }, \mathrm{o} \mathbf{S}_{\mathrm{k}}$ é positivo e, ao contrário dos anteriores, concentra-se nos valores abaixo da $\mathbf{M}_{\mathrm{d}}$. 

Quadro 1 - Evolução da $\mathbf{T}_{\text {min }}, \mathbf{T}_{\text {med }}$ e $\mathbf{T}_{\max } \mathbf{J J A S}$ em Lisboa, durante alguns períodos do século XX.
Table 1 - Evolution of the $\boldsymbol{T}_{\min }, \boldsymbol{T}_{\text {med }}$ and $\boldsymbol{T}_{\max } \boldsymbol{J J A S}$ in Lisbon, during some periods of the XX century.

\begin{tabular}{lccc}
\hline Períodos & $\mathbf{T}_{\min }$ & $\mathbf{T}_{\text {med }}$ & $\mathbf{T}_{\max }$ \\
\hline $1901 / 2001$ & $+1,1$ & $+1,1$ & +2 \\
$1951 / 2001$ & $+1,7$ & $+0,9$ & $+0,1$ \\
$1971 / 2001$ & $+1,8$ & $+1,3$ & $+0,9$ \\
\hline
\end{tabular}

Observando o $\mathbf{K}_{\mathrm{ur}}$, constata-se que as distribuições de todas as temperaturas são muito platicúrticas - com valores heterogéneos - nomeadamente a $\mathbf{T}_{\min }$ que, apesar desta dispersão, obedece aos critérios da distribuição normal, pelos resultados obtidos no teste $\mathbf{S h W}$ e respectivo $p$-value.

Quadro 2 - Estatísticas descritivas da $\mathbf{T}_{\min }, \mathbf{T}_{\text {med }}$ e $\mathbf{T}_{\max }$ JJAS em Lisboa/Geofísico durante o século $\mathrm{XX}$.

Table 2 - Descriptive statistics of the $\mathbf{T}_{\min }, \mathbf{T}_{\text {med }}$ and $\mathbf{T}_{\max } \boldsymbol{J J} \boldsymbol{A S}$ in Lisbon during the $X X$ century.

\begin{tabular}{lcccccccccc}
\hline Temperatura & $\boldsymbol{N}$ & $\mathbf{M}_{\mathbf{d}}$ & $\mathbf{M}_{\mathbf{m}}$ & $\mathbf{M}_{\mathbf{x}}$ & $\mathbf{V}_{\text {ar }}$ & $\mathbf{D}_{\mathbf{p}}$ & $\mathbf{S}_{\mathbf{k}}$ & $\mathbf{K}_{\mathrm{ur}}$ & $\mathbf{S h W}$ & $\boldsymbol{p}$-value \\
\hline $\mathbf{T}_{\text {min }}$ & 101 & 16,969 & 15,500 & 18,575 & 0,435 & 0,659 & 0,141 & $-0,254$ & 0,987 & 0,459 \\
$\mathbf{T}_{\text {med }}$ & 101 & 21,641 & 16,600 & 23,200 & 0,513 & 0,716 & $-0,154$ & $-0,080$ & 0,989 & 0.636 \\
$\mathbf{T}_{\max }$ & 101 & 26,209 & 23,300 & 28,425 & 1,110 & 1,053 & $-0,273$ & 0,022 & 0,989 & 0,585 \\
\hline
\end{tabular}

Ao analisar o comportamento das temperaturas nos últimos 51 anos do século XX (quadro 3), verifica-se um ligeiro aumento na $\mathbf{M}_{\mathbf{d}}$ e também uma diminuição da variabilidade, com excepção da $\mathbf{T}_{\min }$, que sofreu um acréscimo.

$\mathrm{Na}$ estação de Lisboa/Geofísico regista-se, na segunda metade do século XX, um maior peso na distribuição dos valores mais elevados que a $\mathbf{M}_{\mathrm{d}}$. O $\mathbf{S}_{\mathbf{k}}$ nas temperaturas analisadas demonstra uma mudança no sentido negativo na $\mathbf{T}_{\min }$ e $\mathbf{T}_{\text {med }}$, verificando-se, por isso, tendência para a sucessão de temperaturas extremas positivas. $\mathrm{Na} \mathbf{T}_{\max }$, embora ligeira, ocorre o oposto. Na posição da $\mathbf{T}_{\min }$ ao longo da série, observa-se uma maior heterogeneidade, sendo ainda mais platicúrtica comparando com 1901/2001, verifi-

Quadro 3 - Estatísticas descritivas da $\mathbf{T}_{\text {min }}, \mathbf{T}_{\text {med }}$ e $\mathbf{T}_{\text {max }}$ JJAS em Lisboa, 1951/2001.

Table 3 - Descriptive statistics of the $\boldsymbol{T}_{\min }, \boldsymbol{T}_{\text {med }}$ and $\boldsymbol{T}_{\max } \boldsymbol{J} \boldsymbol{J A S}$ in Lisbon, 1951/2001.

\begin{tabular}{lcccccccccc}
\hline Temperatura & $\boldsymbol{N}$ & $\mathbf{M}_{\mathbf{d}}$ & $\mathbf{M}_{\mathbf{m}}$ & $\mathbf{M}_{\mathbf{x}}$ & $\mathbf{V}_{\text {ar }}$ & $\mathbf{D}_{\mathbf{p}}$ & $\mathbf{S}_{\mathbf{k}}$ & $\mathbf{K}_{\mathrm{ur}}$ & $\mathbf{S h W}$ & $\boldsymbol{p}$-value \\
\hline $\mathbf{T}_{\text {min }}$ & 51 & 16,999 & 15,500 & 18,500 & 0,538 & 0,734 & 0,027 & $-0,635$ & 0,981 & 0,584 \\
$\mathbf{T}_{\text {med }}$ & 51 & 21,843 & 20,000 & 23,200 & 0,444 & 0,666 & $-0,391$ & 0,543 & 0,975 & 0,368 \\
$\mathbf{T}_{\max }$ & 51 & 26,677 & 24,447 & 28,425 & 0,541 & 0,735 & $-0,162$ & 0,849 & 0,983 & 0,705 \\
\hline
\end{tabular}


cando-se a ocorrência de valores baixos, mas também muito elevados. Ao invés, os resultados do $\mathbf{K}_{u r}$ na $\mathbf{T}_{m e d}$ e $\mathbf{T}_{\text {max }}$ indicam uma maior concentração dos registos nos valores centrais e por isso são mais mesocúrticas. Todas as distribuições estão dentro da normalidade, segundo o ShW e o seu valor de $p$.

Durante os últimos 31 anos do século transacto (quadro 4) registou-se, em comparação com a segunda metade do século XX, um aumento da $\mathbf{T}_{\text {min }}$ e da $\mathbf{T}_{\text {med }}$ e uma ligeira diminuição da $\mathbf{T}_{\max }$. A variabilidade sofre uma pequena subida em todas as temperaturas analisadas. O comportamento da $\mathbf{S}_{\mathbf{k}}$ é bastante negativo, demonstrando uma importante influência dos valores mais positivos das temperaturas em estudo. Comparando com os últimos 51 anos do século XX, a $\mathbf{T}_{\min }$ é aquela que regista uma maior evolução neste sentido. Observa-se, assim, uma grande concentração de valores mais elevados do que a $\mathbf{M}_{\mathrm{d}}$. Constata-se, igualmente, um menor achatamento em todas as distribuições, apresentando características mesocúrticas com tendência para leptocúrticas, ou seja, menos heterogéneas, sem deixarem de se observar valores extremos - maioritariamente positivos - como demonstram os valores do teste de $\mathbf{S h W}$, pela menor normalidade que apresentam, principalmente na $\mathbf{T}_{\min }$ e $\mathbf{T}_{\text {med }}$. Regista-se uma mudança do regime térmico e uma maior intensidade de calor durante os meses em estudo.

Quadro 4 - Estatísticas descritivas da $\mathbf{T}_{\min }, \mathbf{T}_{\text {med }}$ e $\mathbf{T}_{\max }$ JJAS em Lisboa, 1971/2001. Table 4 - Descriptive statistics of the $\boldsymbol{T}_{\boldsymbol{m i n}}, \boldsymbol{T}_{\boldsymbol{m e d}}$ and $\boldsymbol{T}_{\max }{ }_{\boldsymbol{m}} \boldsymbol{J} \boldsymbol{J} \boldsymbol{A} \boldsymbol{S}$ in Lisbon station, 1971/2001.

\begin{tabular}{lcccccccccc}
\hline Temperatura & $\boldsymbol{N}$ & $\mathbf{M}_{\mathbf{d}}$ & $\mathbf{M}_{\mathbf{m}}$ & $\mathbf{M}_{\mathbf{x}}$ & $\mathbf{V}_{\mathrm{ar}}$ & $\mathbf{D}_{\mathbf{p}}$ & $\mathbf{S}_{\mathbf{k}}$ & $\mathbf{K}_{\mathrm{ur}}$ & $\mathbf{S h W}$ & $\boldsymbol{p}$-value \\
\hline $\mathbf{T}_{\text {min }}$ & 31 & 17,274 & 15,500 & 18,500 & 0,551 & 0,742 & $-0,557$ & $-0,080$ & 0,960 & 0,304 \\
$\mathbf{T}_{\text {med }}$ & 31 & 21,974 & 20,000 & 23,200 & 0,480 & 0,693 & $-0,642$ & 1,320 & 0,947 & 0,129 \\
$\mathbf{T}_{\text {max }}$ & 31 & 26,624 & 24,447 & 28,000 & 0,577 & 0,760 & $-0,516$ & 1,058 & 0,972 & 0,587 \\
\hline
\end{tabular}

Através do quadro 5 podem observar-se as temperaturas mais elevadas do século XX e respectivos anos de ocorrência, na estação de Lisboa/Geofísico.

Verifica-se na $\mathbf{T}_{\min }$ que cinco dos dez anos mais quentes do século precedente ocorreram na década de 90 . Se incluirmos o decénio de 80 , o número sobe para sete. Relativamente à $\mathbf{T}_{\text {med }}$, observa-se que o número de anos quentes nas décadas de $80 \mathrm{e}$ 90 decresce em relação à anterior, somando em conjunto $60 \%$ do total de ocorrências. No entanto, os valores mais baixos encontram-se na $\mathbf{T}_{\max }$, em que apenas três dos dez anos mais quentes do século XX ocorreram a partir de 1980. De realçar que cinco dos dez anos com $\mathbf{T}_{\max }$ mais elevada aconteceram nos anos 40 .

\section{Evolução do número de noites quentes $\left(>20^{\circ} \mathrm{C}\right)$ JJAS em Lisboa}

Na última metade do século XX regista-se uma dinâmica bastante variável do número de noites com temperatura mínima $>20^{\circ} \mathrm{C}$ (fig. 3). Observam-se dois períodos distintos. Um primeiro, entre princípios da década de 50 e finais da de 70, onde não se vislumbra nenhuma tendência em particular, e um segundo, desde 1980 até ao fim da série em análise, em que se constata uma evolução no sentido de um aumento. 
Quadro 5 - Anos mais quentes e respectivas $\mathbf{T}_{\min }, \mathbf{T}_{\text {med }}$ e $\mathbf{T}_{\max }$, JJAS, em Lisboa, durante o século XX, 1901/2001. Os anos e temperaturas a negrito correspondem aos da década de 90 e a sublinhado aos da de 80 .

Table 5-Hottest years and respective $\mathbf{T}_{\min }, \mathbf{T}_{\text {med }}$ and $\mathbf{T}_{\max }, \boldsymbol{J} \boldsymbol{J} \boldsymbol{A S}, n$ Lisbon, during the XX century, 1901/2001. The years and temperatures in bold correspond to the 1990s and the underlined to the 1980s.

\begin{tabular}{rcccccc}
\hline \multirow{2}{*}{ Posição } & \multicolumn{2}{c}{$\mathbf{T}_{\text {min }}$} & \multicolumn{2}{c}{$\mathbf{T}_{\text {med }}$} & \multicolumn{2}{c}{$\mathbf{T}_{\max }$} \\
\cline { 2 - 7 } & Ano & Temp $\left({ }^{\mathbf{0}} \mathbf{C}\right)$ & Ano & Temp $\left({ }^{\mathbf{0}} \mathbf{C}\right)$ & Ano & Temp $\left({ }^{\mathbf{0}} \mathbf{C}\right)$ \\
\hline 1 & 1926 & 18,6 & $\underline{1987}$ & $\underline{23,2}$ & 1962 & 28,4 \\
2 & $\underline{1987}$ & $\underline{18,5}$ & 1949 & 23,1 & 1949 & 28,3 \\
3 & $\underline{1989}$ & $\underline{18,3}$ & $\mathbf{1 9 9 1}$ & $\mathbf{2 3 , 1}$ & 1948 & 28,2 \\
4 & $\mathbf{1 9 9 1}$ & $\mathbf{1 8 , 3}$ & 1926 & 23 & $\underline{1987}$ & $\underline{28}$ \\
5 & $\mathbf{1 9 9 0}$ & $\mathbf{1 8 , 1}$ & $\underline{1989}$ & $\underline{22,9}$ & 1961 & 27,9 \\
6 & 1906 & 18,0 & $\mathbf{2 0 0 0}$ & $\mathbf{2 2 , 9}$ & $\mathbf{2 0 0 0}$ & $\mathbf{2 7 , 9}$ \\
7 & $\mathbf{1 9 9 5}$ & $\mathbf{1 8}$ & $\mathbf{1 9 9 0}$ & $\mathbf{2 2 , 8}$ & 1941 & 27,8 \\
8 & $\mathbf{1 9 9 9}$ & $\mathbf{1 7 , 9}$ & 1961 & 22,7 & $\mathbf{1 9 9 1}$ & $\mathbf{2 7 , 8}$ \\
9 & $\mathbf{2 0 0 0}$ & $\mathbf{1 7 , 9}$ & 1948 & 22,6 & 1944 & 27,5 \\
10 & 1918 & 17,8 & $\underline{1981}$ & $\underline{22,6}$ & 1945 & 27,5 \\
\hline
\end{tabular}

Durante a segunda metade do século XX, o máximo anual de noites quentes JJAS foi de 31 e o mínimo de 0 (quadro 6). Ao observar os registos dos diversos períodos, verifica-se que a $\mathbf{M}_{\mathbf{d}}$ e a variabilidade são mais elevadas no último quartel do século passado. A comparação entre médias através do teste $t$-student demonstra que existem diferenças bastante significativas $(p<0,001)$ entre os períodos 1951/1975 e 1976/2001. Relativamente à $\mathbf{S}_{\mathbf{k}}$, o seu coeficiente nos últimos 51 anos do século XX é muito positivo - influenciado pelos anos com menos noites quentes - acontecendo o mesmo ao analisar apenas o período 1951/1975. Uma distribuição mais simétrica é registada no período 1976/2001, ou seja, observa-se uma diferença em relação a 1951/1975, no sen-

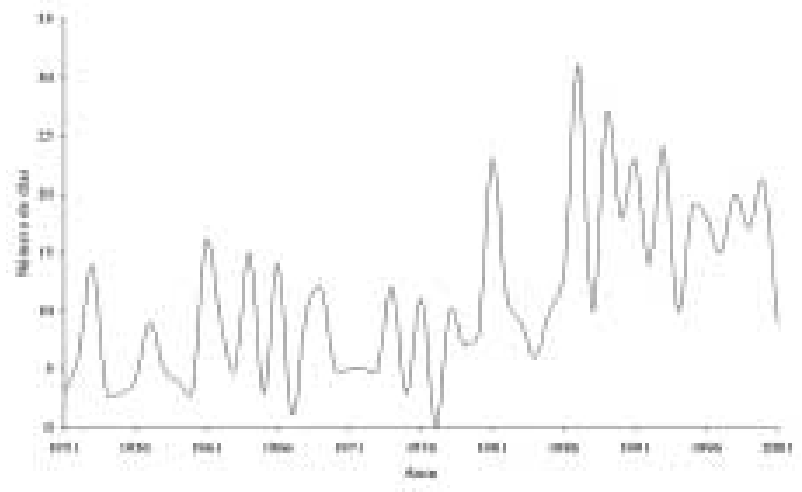

Fig. 3- Número de noites quentes JJAS em Lisboa, 1951/2001.

Fig. 3 - Number of hot nights JJAS in Lisbon, 1951/2001. 
Quadro 6 - Estatísticas descritivas das noites quentes JJAS em Lisboa, durante diferentes períodos da segunda metade do século XX.

Table 6 - Descriptive statistics of the hot nights JJAS in Lisbon, during different periods in the second half of the XX century.

\begin{tabular}{lcrcccccccc}
\hline Temperatura & $\boldsymbol{N}$ & \multicolumn{1}{c}{$\mathbf{M}_{\mathbf{d}}$} & $\mathbf{M}_{\mathbf{m}}$ & $\mathbf{M}_{\mathbf{x}}$ & $\mathbf{V}_{\text {ar }}$ & $\mathbf{D}_{\mathbf{p}}$ & $\mathbf{S}_{\mathbf{k}}$ & $\mathbf{K}_{\text {ur }}$ & $\mathbf{S h W}$ & $\boldsymbol{p}$-value \\
\hline $1951 / 2001$ & 51 & 10,745 & 0 & 31 & 52,55 & 7,249 & 0,836 & 0,213 & 0,934 & 0,007 \\
$1951 / 1975$ & 25 & 6,640 & 1 & 15 & 19,156 & 4,376 & 0,866 & $-0,608$ & 0,845 & 0,001 \\
$1976 / 2001$ & 26 & 14,692 & 0 & 31 & 53,661 & 7,822 & 0,396 & $-0,189$ & 0,967 & 0,554 \\
\hline
\end{tabular}

tido da ocorrência de um maior número de noites com temperatura $>20^{\circ} \mathrm{C}$, nos últimos 25 anos do século XX.

Os resultados da $\mathbf{K}_{u r}$ indicam que há uma maior heterogeneidade na distribuição do número de dias por ano com noites quentes em JJAS. Analisámos toda a série (1951/2001), seguindo-se o período 1976/2001 e, por último, com uma maior homogeneidade, 1951/1975. Exceptuando o período 1976/2001, observa-se que as restantes distribuições não podem ser consideradas normais, devido ao baixo valor de $p(<0,05)$, aproximando-se mais da distribuição log-normal. Comparando as duas curvas (1951/1975 e 1976/2001) (fig. 4), observa-se a tendência da última para uma ocorrência de valores mais elevados, apesar de se verificarem anos com um número reduzido de noites com temperatura $>20^{\circ} \mathrm{C}$.

Analisando a influência de cada mês no total de noites quentes, Julho e Agosto são os que têm mais preponderância, seguidos de Setembro e Junho (fig. 5). Ao comparar o número de noites com temperaturas $20^{\circ} \mathrm{C}$ em JJAS, entre 1951/1975 e 1976/2001, verifica-se que em ambos os períodos estas foram mais elevadas em Julho e Agosto e menores nos restantes meses. Constata-se que em todos os casos, houve um acréscimo importante entre os dois períodos. Entre 1951/1975 e 1976/2001, o número de noites quentes aumentou em Junho, 150\% $(p<0,05)$, em Julho, 108\% $(p<0,05)$, em Agosto, $115 \%(p<0,05)$, e em Setembro 200\% $(p<0,05)$, registando-se uma maior intensi-

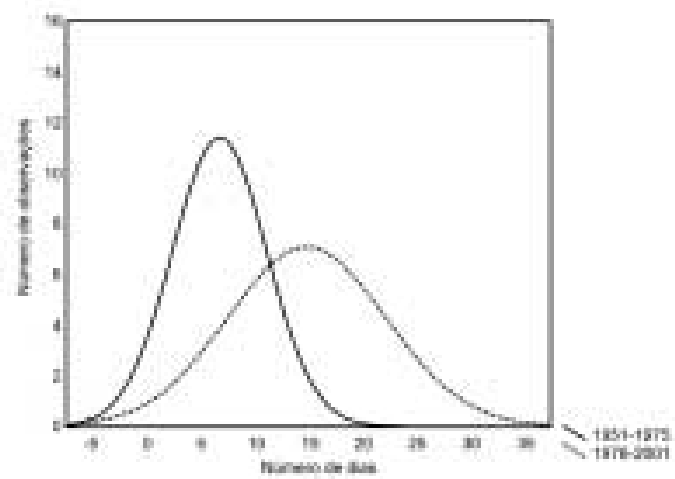

Fig. 4 - Curvas de Gauss da distribuição das noites quentes em diferentes períodos da segunda metade século XX (1951/1975 e 1976/2001) JJAS em Lisboa.

Fig. 4 - Gauss distributions of the hottest nights in different periods of the second half of the XX century (1951/1975 and 1976/2001) JJAS in Lisbon. 
dade na estação quente, através da modificação do seu regime térmico, mas também uma maior extensão do período quente, como comprovam as diferenças observadas no mês de Setembro (fig. 6).
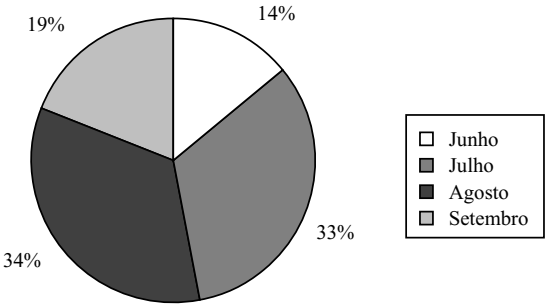

Fig. 5 - Percentagem mensal de noites quentes JJAS, nos últimos 51 anos do século XX em Lisboa.

Fig. 5 - Monthly percentage of hot nights $\boldsymbol{J J A S}$ in the last 51 years of the XX century in Lisbon.

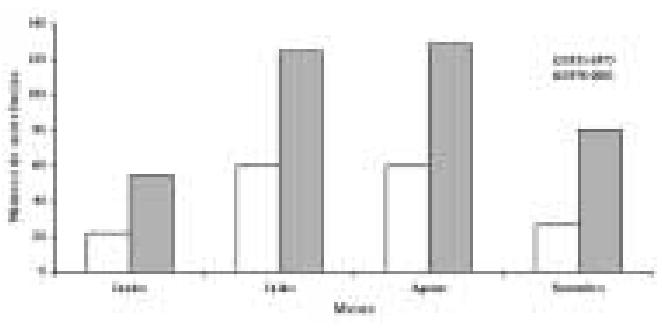

Fig. 6 - Número de noites quentes $\left(>20^{\circ} \mathrm{C}\right)$ JJAS, nos períodos 1951/1975 e 1976/2001 em Lisboa.

Fig. 6-Number of hot nights $\left(>20^{\circ} \mathrm{C}\right) \mathrm{JJAS}$, in the periods $1951 / 1975$ and 1976/2001 in Lisbon.

\section{Evolução da ATD 1951/2001 JJAS em Lisboa}

Desde 1951, nota-se em Lisboa uma tendência para a diminuição da ATD, apesar de alguma variabilidade (fig. 7). Observando os dados dos últimos 51 anos do século XX, verifica-se que a $\mathbf{M}_{\mathrm{d}}$ é quase $10^{\circ} \mathrm{C}$ mais baixa, quando comparada com o período 1951/1975, e mais elevada em relação a 1976/2001 (quadro 7). Aplicando um teste $t$-student, na relação entre 1951/1975 e 1976/2001, registam-se diferenças bastante significativas, com um $p<0,001$. O comportamento da $\mathbf{S}_{\mathbf{k}}$ 1951/2001 demonstra uma assimetria negativa, por isso ligeiramente influenciada pelos valores mais elevados da série, verificando-se a mesma dinâmica no período 1976/2001. Entre 1951/1975 regista-se uma forte influência dos valores mais baixos enquanto que ao observar a $\mathbf{K}_{\mathbf{u r}}$, para o mesmo espaço de tempo, constata-se a presença de uma curva muito leptocúrtica, ou seja, uma maior concentração. As restantes séries apresentam valores mais heterogéneos na distribuição, nomeadamente durante os últimos 26 anos do século XX.

Quadro 7 - Estatísticas descritivas da evolução da ATD JJAS em diferentes períodos da segunda metade do século XX em Lisboa.

Table 7 - Descriptive statistics of the DTR evolution $\boldsymbol{J J A S}$ during different periods of the second half of the XX century in Lisbon.

\begin{tabular}{lcccccccccc}
\hline Temperatura & $\boldsymbol{N}$ & $\mathbf{M}_{\mathbf{d}}$ & $\mathbf{M}_{\mathbf{m}}$ & $\mathbf{M}_{\mathbf{x}}$ & $\mathbf{V}_{\mathrm{ar}}$ & $\mathbf{D}_{\mathbf{p}}$ & $\mathbf{S}_{\mathbf{k}}$ & $\mathbf{K}_{\mathrm{ur}}$ & $\mathbf{S h W}$ & $\boldsymbol{p}$-value \\
\hline $1951 / 2001$ & 51 & 9,678 & 8,3 & 11,525 & 0,441 & 0,664 & $-0,146$ & 0,320 & 0,964 & 0,129 \\
$1951 / 1975$ & 25 & 10,04 & 8,947 & 11,520 & 0,254 & 0,504 & 0,577 & 2,349 & 0,936 & 0,125 \\
$1976 / 2001$ & 26 & 9,32 & 8,300 & 10,500 & 0,376 & 0,613 & $-0,107$ & $-0,727$ & 0,966 & 0,540 \\
\hline
\end{tabular}




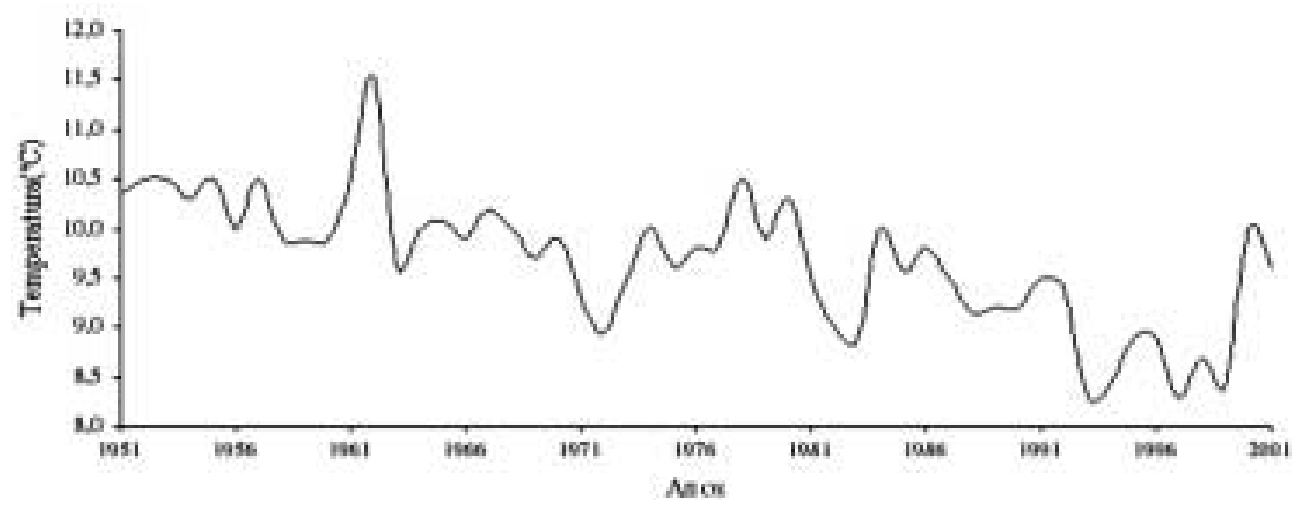

Fig. 7 - Evolução da ATD nos últimos 51 anos do século XX, JJAS em Lisboa. Fig. 7 - Evolution of the DTR during the last 51 years $\boldsymbol{J J A S}$ in Lisbon.

Relativamente à normalidade das distribuições, observa-se que é maior entre 1976/2001 e menor, com valores de $p$ muito próximos, entre 1951/2001 e 1951/1976. Entre 1951/2001, devido à ligeira influência dos valores mais elevados e, entre 1951/1976, dos mais baixos. Visualizando a figura 8 , comprova-se a tendência para a diminuição da ATD no último quartel do século XX. No entanto, durante o mesmo período não deixaram de se observar valores elevados de ATD.

A dinâmica do número de noites com temperatura $>$ a $20^{\circ} \mathrm{C}$ demonstra uma tendência inversa à ATD, como indica a figura 9. Apesar da correlação entre ambas ser de 0,393 e um $\mathrm{r}^{2}$ de apenas 0,154 , é significativo um valor de $p<0,01$, encontrandose todos os valores dentro de um intervalo de confiança de $99 \%$.

\section{DISCUSSÃO}

São diversos os estudos que apontam para um aquecimento global durante o século XX, para o qual a expansão das cidades e as respectivas IC têm contribuído, apesar desta influência não estar suficientemente explicada. Nos últimos 100 anos registou-se um aumento da temperatura média a nível global de $0,6^{\circ} \mathrm{C}$ e na Europa de $0,95^{\circ} \mathrm{C}$. Estima-se que o século XX foi o mais quente dos últimos 1000 anos (IPCC, 2001; Karoly et al., 2003; AEA, 2004; Meehl e Tebaldi, 2004). Em Lisboa, a IC apresenta uma intensidade média de $2,5^{\circ} \mathrm{C}$, dependendo a sua configuração espacial dos regimes de vento e da situação sinóptica. A intensidade média nocturna - calculada a partir da diferença entre a temperatura da cidade e um ponto nos arredores - é maior do que noutras cidades da Península Ibérica, como Salamanca $\left(1,3^{\circ} \mathrm{C}\right)$ (Alonso et al., $2003)$ e Granada $\left(2^{\circ} \mathrm{C}\right)$ (Montálvez et al., 2000), e menor do que em Barcelona $\left(2,9^{\circ} \mathrm{C}\right.$ ) (Moreno, 1994).

Ao longo do século XX a $\mathbf{T}_{\min }$ de $\mathbf{J J A S}$ em Lisboa aumentou progressivamente até ao final da década de 30 , sofrendo posteriormente um novo incremento a partir dos anos 70, desta vez de maior magnitude. Observa-se que existe uma tendência geral dos valores acima da média das temperaturas e consequentemente de valores extremos, principalmente nos últimos 31 anos. Relativamente à $\mathbf{T}_{\text {med }}$, a dinâmica registada 


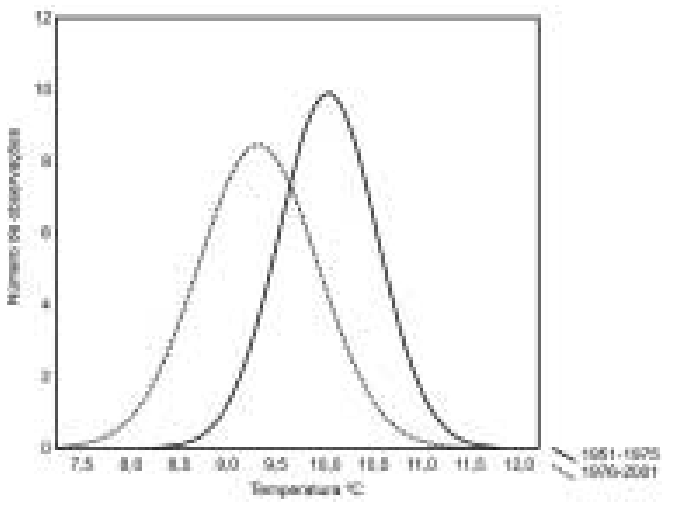

Fig. 8 - Curvas de Gauss para a distribuição da ATD em diferentes períodos da segunda metade do século XX (1951/1975

e 1976/2001) JJAS em Lisboa.

Fig. 8 - Gauss distributions for the DTR during different periods

in the second half of the XX century $(1951 / 1975$ and 1976/2001)

JJAS in Lisbon.

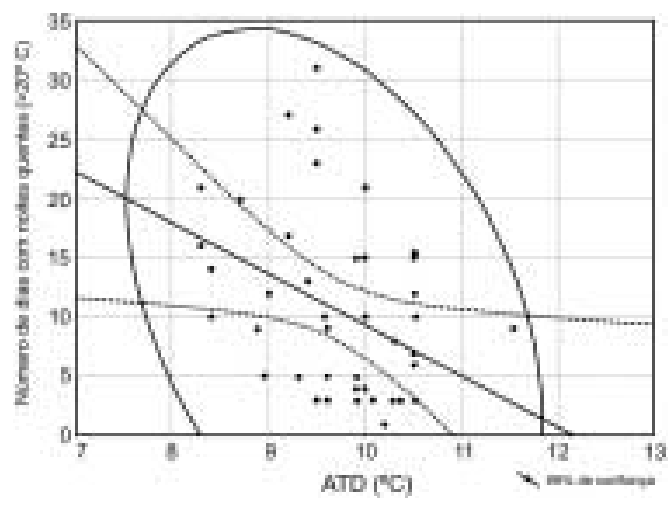

Fig. 9 - Correlação entre o número de dias com noites quentes e ATD JJAS em Lisboa.

A elipse limita o perímetro do intervalo de confiança a $99 \%$.

Fig. 9 - Correlation between the number of days with hot nights and DTR JJAS in Lisbon. The elipse show the confidence interval at $99 \%$.

é semelhante; no entanto, o último período de análise foi marcado por uma maior variabilidade do que a mínima. $\mathrm{O}$ grande aumento de $\mathbf{T}_{\max }$ registou-se nos primeiros 50 anos do século XX, nomeadamente no período 1940/1950, descrevendo uma tendência negativa até 1981 e observando-se posteriormente uma ligeira tendência positiva no período 1971/2001. Ao longo de todo o século, a $\mathbf{T}_{\max }$ é a que sofre maior aumento, embora registe uma subida mais reduzida depois de 1950, apesar de ocorrerem valores bastante positivos desde 1980. Os resultados obtidos estão de acordo com algumas investigações efectuadas sobre a evolução da temperatura estival na Europa durante o século XX. Moberg e Jones (2005), ao estudarem a evolução da temperatura de Junho, Julho e Agosto (JJA) na Europa ao longo do século XX, identificaram igualmente uma tendência de aquecimento entre 1901/1999 e 1921/1999. Os autores verificaram que a $\mathbf{T}_{\max } 1901 / 1950$ sofreu um substancial aumento, sendo menor que na segunda metade do século $X X$, registando-se o inverso na $\mathbf{T}_{\min }$ para a qual observaram, no período 1946/1999, uma subida. Domokos et al. (2003) registaram ao longo do século XX (1901/98) uma tendência para um acréscimo da temperatura no centro e Sul da Europa durante o Verão, observando igualmente uma grande frequência de eventos quentes nos finais dos anos 30, 40 e na última década do século XX. Em Portugal, Miranda et al. (2002) investigaram o comportamento da $\mathbf{T}_{\min }$ e $\mathbf{T}_{\max }$ estival em Lisboa durante o século XX, apenas durante os meses de JJA, observando uma maior tendência de aumento na $\mathbf{T}_{\min }$ do que na $\mathbf{T}_{\max }$.

$\mathrm{O}$ padrão de noites quentes JJAS, na última metade do século $\mathrm{XX}$, demonstra a existência de duas dinâmicas diferentes, entre a década de 50 e finais de 70 - sem tendência - e o período posterior a 1980 - com tendência positiva. Ao estabelecer a comparação entre 1951/1975 e 1976/2001, identificam-se diferenças bastante significativas, 
que também são visíveis na comparação entre a mediana de cada mês, principalmente em Setembro. Verificam-se aumentos na $\mathbf{M}_{\mathbf{d}}$ e da $\mathbf{V}_{\mathrm{ar}}$, característicos de uma tendência para uma alteração do padrão climático (comunicação pessoal de Martin-Vide, 2007). Outros estudos também indicam a maior ocorrência de noites quentes durante o século XX. Moberg e Jones (2005) identificam uma tendência para um incremento de noites quentes entre 1901/1999 na Europa. Frich et al. (2002) estudaram os extremos climáticos em todo o globo entre 1946/1999 e concluem igualmente que há um aumento estatisticamente significativo de noites quentes.

Observa-se em Lisboa, durante a última metade do século em JJAS, uma tendência para a diminuição da ATD, principalmente pelo aumento mais rápido da $\mathbf{T}_{\min }$ em relação à $\mathbf{T}_{\max }$. A comparação entre 1951/1975 e 1976/2001 revela diferenças significativas. Esta tendência negativa $\left(-0,79^{\circ} \mathrm{C}\right)$ já fora identificada ao nível global para JJA 1953/1993 (Easterling et al., 1997), e igualmente em algumas cidades. Balling et al. (1990), ao estudarem o impacto da $\mathbf{T}_{\max }$ e $\mathbf{T}_{\text {min }}$ na $\mathbf{T}_{\text {med }}$ durante a segunda metade do século XX em JJA em Phoenix, demonstram que a $\mathbf{T}_{\text {med }}$ subiu mais do que a $\mathbf{T}_{\max }$, e a existência de mais dias com $\mathbf{T}_{\min }$ extremas do que $\mathbf{T}_{\max }$, o que pode ser uma consequência da IC (Tarleton e Katz, 1995). No entanto, esta dinâmica não é igual em todas as cidades. Unkasevic et al. (2005) investigaram a tendência das temperaturas extremas em Belgrado em JJA 1975/2003 e constataram que a $\mathbf{T}_{\max }$ sofreu um maior incremento que a $\mathbf{T}_{\min }$.

Apesar da relação inversa do número das noites com temperaturas $>20^{\circ} \mathrm{C}$ e da ATD e da significância da sua correlação, não parece haver uma correlação muito forte entre ambas as variáveis e os seus comportamentos, ainda que o aumento da $\mathbf{T}_{\min }$ tenha uma influência fundamental.

\section{CONCLUSÕES}

Apesar dos diferentes ritmos temporais, observa-se um aumento generalizado das temperaturas na estação meteorológica do centro da cidade de Lisboa em JJAS - principalmente da $\mathbf{T}_{\min }$ no último quartel do século XX - para o qual a IC, o crescimento pouco ordenado da cidade, que impede a penetração do vento de Norte durante o Verão e o aquecimento global poderão ter influência. Deve ter-se, em conta que a estação se encontra no Jardim Botânico e que podem existir diferenças significativas entre este e o bairro limítrofe (Alcoforado, 1992). No entanto, segundo Lopes (2003) e Alcoforado e Lopes (2003), a expansão da cidade reduziu a velocidade média do vento entre 26 a $30 \%$ e poderá estar implicada no comportamento das temperaturas, contribuindo também para uma maior acumulação de poluentes na cidade. Segundo os resultados observados neste trabalho - e outros referidos - verifica-se um aumento da temperatura, do número de noites quentes e diminuição da ATD.

Como conclusões gerais do estudo constata-se:

- Um aumento importante da $\mathbf{M}_{d}$ e uma tendência para o incremento de valores extremos positivos da $\mathbf{T}_{\text {min }}$, principalmente nos últimos 31 anos do século XX, com especial relevância a partir de 1980.

- Um ligeiro acréscimo da $\mathbf{M}_{\mathrm{d}} \mathrm{e}$ da variabilidade na $\mathbf{T}_{\text {med }}$. Como na $\mathbf{T}_{\text {min }}$, na $\mathbf{T}_{\text {med }}$ observa-se uma tendência para a ocorrência de valores mais elevados a partir de 1971, nomeadamente nos últimos 20 anos do século XX. 
- Um grande aumento da $\mathbf{T}_{\max }$ 1901/1950, principalmente entre $1940 / 50$, em que se observa uma grande parte dos anos mais quentes de todo o século. Regista-se uma diminuição da $\mathbf{M}_{\mathbf{d}}$ 1971/2001 em comparação com os outros períodos de análise, apesar de existirem anos muito quentes.

- A existência de dois padrões distintos na evolução do número de noites quentes, nos períodos 1951/1975 e 1976/2001, a primeira sem tendência marcada e a segunda com tendência positiva, revela diferenças bastante significativas, que podem expressar a evolução para uma nova dinâmica, para um novo regime térmico e para o aumento da intensidade da estação quente.

- Uma extensão da estação quente, registada pelo grande número de noites quentes em Setembro, principalmente no período 1976/2001, justifica a sua inclusão nos meses de Verão, o que não acontece, por norma, em muitos estudos.

- Uma diminuição da ATD, principalmente devido ao aumento da $\mathbf{T}_{\min }$, no último quartel do século XX. Uma alteração estatisticamente significativa no comportamento dos períodos 1951/1975 e 1976/2001, com uma diferença negativa do primeiro para o segundo, apesar de este ser ainda ligeiramente condicionado pelos valores superiores à $\mathbf{M}_{\mathbf{d}}$.

- Não existe uma relação muito forte entre a dinâmica do número de noites quentes e da ATD.

\section{AGRADECIMENTOS}

Os autores agradecem a Joan Albert López-Bustins e a Arturo Sánchez Lorenzo as sugestões importantes na elaboração da metodologia deste trabalho. Ao Prof. Dr Javier Martin-Vide, pela dissipação de algumas dúvidas relacionadas com as alterações climáticas e clima urbano. À Prof. Dra Maria João Alcoforado, pelo pronto e rápido auxílio sobre alguns aspectos do clima urbano de Lisboa e a dois revisores anónimos, pelas pertinentes sugestões na organização do trabalho.

\section{BIBLIOGRAFIA}

Agência Europeia do Ambiente (AEA) (2004) Sinais Ambientais 2004. [Acedido de acordo com as normas em 13 de Maio de 2006]. http://europa.eu.int

Akbari H, Pomerantz M, Taha H (2001) Cool surfaces and shande trees to reduce energy use and imprive air quality in urban areas. Solar Energy, 70 (3): 2975-310.

Alcoforado, M J, Andrade, H (2007) An international perspective on the interaction between humans and nature. In Marzluff J M, Endlicher W (eds.) Urban Ecology. Seattle (in press).

Alcoforado M J, Andrade H (2006) Nocturnal urban heat island in Lisbon (Portugal): main features and modelling attempts. Theor. Appl. Climatol, 84 (1-3): 151-159.

Alcoforado M J, Lopes H (2003) Wind fields and temperature patterns in Lisbon (Portugal) and their modification due to city growth. Fifth International Conference on Urban Climate, Lodz, Poland, Vol 1: 383-386.

Alcoforado M J (1992) - O Clima da Região de Lisboa. Contrastes e Ritmos Térmicos. Memórias do Centro de Estudos Geográficos, vol. 15. 
Alonso M S, Labajo J L, Fidalgo M R (2003) Characteristics of urban heat island in the city of Salamanca. Atmósfera, Vol 16 (3) : 137-148.

Balling R C, Skindlow J A, Phillips D A (1990) The impact of increasing summer mean temperatures on extreme maximum and minimum temperatures in Phoenix, Arizona, J. Climate, 3 : 1491-1494.

Bosch X (2003) European heatwave causes misery and deaths. The Lancet, 362: 543.

Bradley R S (2000a) Past global changes and their significance for the future. Quaternary Science Reviews, 19: 391-402.

Bradley R S (2000b) 1000 Years of Climate Change. Science, 288: 1353-1355.

Bretz S, Akbari H, Rosenfeld A (1998) Practical issues for using solar reflective materials to mitigate urban heat islands. Atmospheric Environment, 32 (1): 95-101.

Chimklai P, Hagishima A, Tanimoto J (2004) A computer system to suport Albedo calculation in urban areas. Building and Environment, 39: 1213-1221.

Domokos P, Kysel_J, Piotrowicz K, Petrovic P Likso T (2003) Variability of extreme temperature events in South-Central Europe During the $20^{\text {th }}$ century and its relatrionship with large-scale circulation, Int. Journal Climatology, 23: 987-1010.

Easterling D R, Horton B, Jones P D, Peterson T C, Karl T R, Parker D E, Salinger m J, Razuvayev V, Plummer N, Jamason P, Folland K C (1997) Maximum and minimum temperature trends for the globe, Science, 277: 364-367.

EPA (2003) Cooling summertime temperatures, strategies to reduce urban heat island. [Acedido em 13 de Maio de 2006]. http://www.epa.gov/heatisland/resources/pdf/HIRIbrochure.pdf

Epstein P R, Rogers C (2004) Inside the Greenhouse, the impacts of $\mathrm{CO}_{2}$ and climate change on public health in the inner city. Report from Center for Health and Global Environment Harvard medical School: 28.

Frich P, Alexander L V, Della-Marta P, Gleason B, Haylock M, Klein Tank A M G, Peterson T (2002) Observed coherent changes in climatic extremes during the second half of the twentieth century. Climate Research, 19: 193-212.

Fukuoka Y (1997) Biometeorological studies on urban climate. Int. Journal Biometeorology, 40: 54-57.

Hinkel K M, Nelson F E, Klene A E, Bell J E (2003) The urban heat island in winter at Barrow, Alaska. Int. Journal Climatology, 23: 1889-1905.

IPCC (2001) Cambio Climático, Resumén para Responsables de Políticas: 38. [Acedido em 13 de Maio de 2006]. http://www.ipcc.ch

Jin M, Dickinson R E, Zhang D A (2005) The footprint of urban areas on global climate as characterized by MODIS. Journal of Climate, 18: 1551-1565.

Kalnay E, Cai M (2003) Impact of urbanization on climate. Nature, 432: 528-531.

Karoly D J, Braganza K, Stott P A, Arblaster J M, Meehl G A, Broccoli A J, Dixon K W (2003) Detection of a human influence on a North American climate. Science, 302: 1200-1202.

Klein Tank A M G, Können G P, Selten F M (2005) Signals of anthropogenic influence on European warming as seen in the trendpatterns of daily temperature variance. Int. Journal Climatology, 25: $1-16$.

Klein Tank A M G, Wijngaard J B, Könen G P, Böhm R, Demarée G, Gocheva A, Mileta M, Pashiardis S, Hejkrilk L, Kern-Hansen C, Heino R, Bessmoulin P, Müllerr-Wetermeir G, Tzanakou M, Szalai S, Páldóttir T, Fitzgerald D, Rubin S, Capaldo M, Maugeri M, Leitass A, Bukantis A, Aberfeld R, Vanengelen A F V, Forland E, Mietus M, Coelho F, Mares C, Razuvaev V, Nieplova 
E, Cegnar T, Antonio López J, Dahlström B, Moberg A, Kirchhofer W, Ceylan A, Pachaliuk O, Alexander L V, Petrovic P (2002) Daily dataset of 20th century surface air temperature and precipitation series for the european climate assessment, Int. Journal Climatology, 22: 1441-1453.

Landsberg H (1973) The meteorological utopian city. Bulletin American Meteorological Society, 54 (2): 86-89.

Lopes A (2003) Modificações no clima urbano de Lisboa como consequência do crescimento urbano, vento, ilha de calor de superfície e balanço energético. Dissertação de doutoramento, Universidade de Lisboa, Lisboa.

Lowry W P (1976) El clima de las ciudades. Selecciones de Scientific American - La ciudad, Herman Blume, Madrid: 160-169.

McClatchey J (2005) Regional and local climates. In Holden J. (ed.) An introduction to Physical Geography and the Environment. Prentice Hall, Edinburgh: 80-96.

Meehl G A, Tebaldi C (2004) More intense, more frequent, and longer lasting feat waves in the $21^{\text {st }}$ Century. Science, 305: 994-997.

Miranda P, Coelho F E, Tomé A R, Valente M A, Carvalho A, Pires C, Pires H O, Pires V C. Ramalho C (2002) 20th Century portuguese climate and climate cenarios. In Santos F D, Forbes K, Moita R (ed.) Climate Change in Portugal, Scenarios, Impacts and Adaptations Measures. SIAM Project, Lisboa: 28-83.

Moberg A, Jones P D (2005) Trends in indices for extremes in daily temperature and precipitation in Central and Western Europe, 1901-99, Int. Journal Climatology, 25: 1149-1171.

Montálvez J P, Rodriguez A, Jiménez J I (2000) A study of the urban heat island of Granada. Int. Journal Climatology, 20: 899-911.

Moreno M C (1994) Intensity and form of urban heat island in Barcelona. Int. Journal Climatology, 14: 705-710.

Moreno M C, Martin Vide J (2002) Algunas ideas propias de la climatología urbana de cara a la planificación urbana y rural. Aportaciones Geográficas en Memoria del Prof. L. Miguel Yetano Ruiz: 395-400.

Oke T R (1987) Boundary Layer Climates, $2^{\text {nd }}$. Methuen, London, 435 p.

Parker D E (2004) Large-scale warming is not urban. Nature, 432: 290.

Prado R T A, Ferreira F L (2005) Measurement of albedo and analysis of its influence in the surface temperature of building roof materials. Energy and Buildings, 37: 295-300.

Sala J Q, Olcina G, Cuevas A P, Cantos J O, Amoros A R, Chiva E M (2000) Climatic warming in the spanish mediterranean: natural trend or urban effect. Climate Change, 46: 473-483.

Scherrer S C, Appenzeller C, Liniger M A (2006) Temperature trends in Switzerlandand Europe: Implications for climate nomals, Int. Journal Climatology, 26: 565-580.

Shapiro S, Wilk M (1965) An analysis of variance test for normality. Biometrika, 52: 591-611.

Shar C, Jendritzky G (2004) Hot news from summer 2003. Nature, 432: 559-560.

Solecki W D, Rosenzweig C, Pope G, Clark M, Lazar V (2002) The urban heat island in the greater Newark and Camden regions of New Jersey: current and future dimensions. Presentation in The Conference, Urban Heat island Summit, Toronto.

Stone D A, Weaver A J (2002) Daily maximum and minimum temperature trends in climate model. Geoph. Rea. Let., 29 (9-10): 1029-2001.

Taha H (1997) Urban climates and heat islands: albedo, evapotranspiration and anthropogenic heat. Energy and Buildings: 25: 99-103. 
Tarleton L F, Katz R (1995) Statistical explanation for trends in extreme summer temperatures at Phoenix, Arizona. Journal of Climate, 8: 1704-1708.

Unkasevic M, Vujovic D, Tosic I (2005) Trends in extreme summer temperatures at Belgrade. Theor. Appl. Climatol., 82, 199-205.

Vogt J A, Oke T R (2003) Thermal remote sensing of urban climates. Remote Sensing of Environment, 86: $370-384$.

Vose R S, Easterling D R, Gleason B (2004) Maximum and minimum trends for the globe; an update to 2004. [Acedido em 13 de Maio de 2006]. http://ams.confex.com/ams/pdfpaper/100744.pdf 